\title{
Ética y medicina en la obra de Galeno
}

\author{
Rosa M. Moreno Rodríguez (*) \\ (*) Departamento de Anatomía Patológica e Historia de la Ciencia. Universidad de Granada \\ rmoreno@ugr.es
}

Dynamis

[0211-9536] 2013; $33(2): 441-460$
Fecha de recepción: 17 de abril de 2012

Fecha de aceptación: 13 de enero de 2013

SUMARIO: 1.-Introducción. 2.-Filosofía y medicina. 3.-Doctrina moral. 4.-Medicina y ética.

RESUMEN: En este trabajo propongo que la moral galénica ha de entenderse como parte fundamental de la doctrina médica. Recientes publicaciones que relacionan el proyecto existencial de Galeno con la metodología médica y la apología de Hipócrates, revelan la idoneidad de esta perspectiva: que para Galeno, el saber médico moldea la definición de la buena vida, es un recurso seguro para conseguir la excelencia y una de las tareas de la medicina. La hipótesis básica es que la filosofía práctica de Galeno se sustenta en la idea de que las actividades con significado moral (apetitos y emociones) tienen un cometido en el mantenimiento del organismo. Así se justifica la introducción de la medicina en el ámbito del comportamiento humano: si las emociones y las pasiones tienen un papel fisiológico, su explicación incumbe a la medicina, la cual también realiza el diagnóstico e indica en su caso el tratamiento, siguiendo el procedimiento teórico propuesto para cualquier tipo de disfunción. Además, una idea tradicional de enfermedad, que la entiende como desequilibrio entre las partes, hace de la racionalidad una guía segura para la formación del carácter, ya que permite explicar la conducta viciosa sin involucrarla. Esta concepción autorizó a Galeno, y a la medicina, a intervenir en el debate en torno a la recurrente crisis social del mundo romano, con mayor beneficio, según sus propias palabras, que el ofrecido por la filosofía. Al proponer una interpretación de la angustia existencial, del sufrimiento y de la persistencia del mal como un desequilibro entre las partes orgánicas, aludía también al quebrantamiento de la estructura jerarquizada del cuerpo, y suscitaba la creencia de que el ambiente reprobable tenía su causa en el desorden de la disposición social.

PALABRAS CLAVE: Galeno, ética médica, psicología y moral, virtud y dieta galénica, paradigma hipocrático.

KEY WORDS: Galen, medical ethics, psychology and morality, virtue and health regimen, Hippocratic moral guide. 


\section{Introducción}

El resurgido interés en el estudio de la obra de Galeno descansa en la polimatía que desplegó durante su vida ${ }^{1}$. Ese conjunto de intereses diversos ofrece una representación de la creación y la constitución del conocimiento en el mundo romano y de la propia doctrina médica ${ }^{2}$. Su estilo, que había sido calificado de pretencioso y pedante, es apreciado ahora en su función retórica: Galeno lo habría utilizado como investidura togal para participar en los debates públicos intelectuales, afirmando su dedicación médica ${ }^{3}$. Es decir, habría pretendido que la medicina pudiese intervenir en el conocimiento y tratamiento de los asuntos humanos a la par que la filosofía. Se destaca el uso que hizo de la doctrina biológica aristotélica para realizar esta tarea, como fundamento de la metodología médica en un marco estocástico pero eficaz, también, en la resolución del estatuto epistémico de los particulares en el seno del conocimiento científico ${ }^{4}$. Este esfuerzo teorético hace de Galeno un pensador de la talla de Ptolomeo y Arquímedes, Platón y Aristóteles o Alejandro de Afrodisias ${ }^{5}$. Además, su figura puede quedar definitivamente ennoblecida al enmarcar este propósito en el desprestigio que afectaba la medicina, según consideró al llegar por primera vez a Ro$\mathrm{ma}^{6}$. Afirmaba que la incompetencia de los médicos era absoluta debido a la corrupción general del ambiente social: pervertidos los médicos por la

1. König, Jason Peter. Conventions of prefatory self-presentations in Galen's On the order of my own books. In: Gill, Chistopher; Whitmarsch, Tom; Wilkins, John, eds. Galen and the world of knowledge. Greek culture in the Roman world. Cambridge: Cambridge University Press; 2009, p. 35-58 (35-39); véase también en este libro el capítulo de Staden, Heinrich von. Staging the past, staging oneself: Galen on Hellenistic exegetical traditions (p. 132-156). Nutton, Vivian. The fortunes of Galen. In: Hankinson, Richard, ed. The Cambridge companion to Galen. Cambridge: Cambridge University Press; 2008, p. 355-390.

2. Hankinson, Richard J. The man and his work. In: Hankinson, n. 1, p. 1-33; Nutton, Vivian. Galen's library. In: Gill; Whitmarsch; Wilkins, n. 1, p. 19-34 (23-34).

3. García Ballester, Luis. Galen's medical works in the context of his biography. In: Arrizabalaga, Jon et al., eds. Galen and galenism. Theory and medical practice from Antiquity to the European Renaissance. Aldershot: Ashgate Variorum; 2002, p. 1-53 (42-46); Tieleman, Teun. Galen and the stoics, or: the art of not naming. In: Gill; Whitmark; Wilkins, n. 1, p. 282-299 (286-289).

4. Tieleman, Teun. Methodology. In: Hankinson, n. 1, p. 49-65 (49-54); Eijk, van der Phillip. Therapeutics. In: Hankinson, n. 1, p. 283-303 (286); Boudon-Millot, Véronique. Art, science et conjecture chez Galien, In: Barnes, Jonathan; Jouanna, Jacques, eds. Galien et la philosophie. Geneve: Vandoeuvres; 2002, p. 269-305 (283-298).

5. Flemming, Rebecca. Commentary. In: Hankinson, n. 1, p. 323-354 (329).

6. Nutton, n. 1, p. 363. 
ambición de riqueza, habían olvidado las enseñanzas de Hipócrates y se dedicaban a la complacencia de sus pacientes; éstos, «ricos y potentados, vivían inmersos en los placeres del lujo, en la tryphé platónica, y se comportaban como bestias llevados por el goce y la riqueza» ${ }^{7}$.

A modo de hermenéutica, la reinterpretación de la obra galénica nos ofrece una imagen de la mentalidad cultural, en la que la agencia de las aspiraciones personales se valora como un componente fundamental de la creación del conocimiento en la Roma imperial. Por esto, las fuentes utilizadas por Galeno y el análisis de su actividad clínica y dialéctica confluyen para establecer el contexto heurístico fundamental; es decir, el estudio de la relación entre vida y método, como aparece formulada por Véronique Boudon-Millot: «vida y método obedecen necesariamente las mismas reglas y necesariamente usan las mismas herramientas: experimento y razonamiento» ${ }^{8}$.

Este trabajo, dedicado al estudio de la doctrina ética de Galeno, intenta continuar dicho tipo de análisis, pero se centra en las ideas médicas en torno a la especie humana y su desarrollo en el marco de las diversas formas de la naturaleza paradigmática y generatriz. Se mantiene en él la imposibilidad de separar la ciencia de la axiología, por eso se expone la doctrina médica como fundamento de un pensamiento acerca de qué es la vida buena ${ }^{9}$, de

7. Tomado de Vegetti, Mario. Galeno e la rifondazione della medicina. Dynamis. 1995; 15: 67-101 (68). Si en la referencia inicial no se indica lo contrario, todas las traducciones castellanas son mías incluyendo las versiones de las obras galénicas.

8. Boudon-Millot, Véronique. Galen's Bios and Methodos: from ways of life to path of knowledge. In: Gill; Whitmarsh; Wilkins, n. 1, p. 175-189 (181).

9. En este trabajo, utilizo el sistema de citas propuesto por Hankinson en la edición Cambridge Companion to Galen (n. 1, p. xix-xxi): las abreviaturas son las indicadas en esa obra (p. 391 397); cuando la contiene, ofrezco la referencia según la edición de C. G. Kühn (C. Galeni Opera Omnia, 20 vols. Leipzig; 1821-1830 (reimpr. Hildesheim; 1964), seguida de las ediciones manejadas [= colección o autores]; por último, conservo la información sobre las líneas tras las páginas del texto griego. Detallo a continuación la lista de obras con sus títulos completos en latín, seguidas de las ediciones críticas y las abreviaturas correspondientes:

Adhortatio ad artes addiscendas: K. I, 1-39 (Protr.). De proprium animi cuiuslibet affectuum dignotione et curatione: K. V, 1-57. De ciuiusque animi peccatorum dignotione et curatione. K. V, 58-103. Edición: Elst Robert van der. Traité des passions de l'âme et de ses erreurs par Galien. Paris: CH. Delagrave; 1914 (Aff. Dig.= Elst; Pecc. Dig. = Elst). Quod animi mores corporis temperamenta sequuntur: K. IV, 767-822. Edición García Ballester, Luis. Alma y enfermedad en la obra de Galeno. Traducción y comentario del escrito Quod animi mores corporis temperamenta sequuntur. Granada: Publicaciones Universidad de Granada; 1972 (QAM = García Ballester). De indolentia: Boudon-Millot, Véronique; Jouanna, Jacques. Galien. Tome IV. Ne pas se chagriner. Paris: Les Belles Lettres; 2010 (Ind. = Boudon-Millot; Jouanna). Quod 
cómo se ha de concebir y de cómo llevar una existencia que conduzca a la vida buena ${ }^{10}$.

Se tratará, en primer lugar, de argumentar cómo Galeno consideró insuficiente un acercamiento propiamente filosófico, según la defensa que hizo de Hipócrates como pedagogo y modelo. Se describirá después su doctrina moral, y se terminará estudiando la ética como base de su proyecto legitimador de la medicina griega en la sociedad romana.

\section{Filosofía y medicina}

La iniciativa galénica de incorporar la filosofía al estudio de la medicina ha recibido un considerable número de interpretaciones, casi siempre a favor de considerar la primera como fuente de recursos epistémicos, éticos y deontológicos ${ }^{11}$. Según Lloyd, con la filosofía Galeno habría podido subsanar una carencia epistemológica y adquirir una formación moral, que podía haber tenido fines clientelares: «sus pacientes debían apreciar que él se dedicaba al arte de la medicina por el bien de la humanidad, no sólo por dinero» ${ }^{12}$.

El propio Galeno justificó el aspecto moral en un pequeño tratado, Quod optimus medicus sit quoque philosophus: la medicina es un arte filantrópico y los médicos, dada la destreza y dedicación que han de guardar

optimus medicus sit quoque philosophus: K. I, 53-63. Edición: Boudon-Millot, Véronique. Galien: Introduction générale; Sur l'ordre de ses propres livres; Sur ses propres livres; Que l'excellent médecin est aussi philosophe. Paris: Les Belles Lettres; 2007 (Opt. Med. = Boudon-Millot). De placitis Hippocratis et Platonis: K, V, 181-805. Edición: De Lacy, Phillip. On the Doctrines of Hippocrates and Plato, CMG V, 4,1,2. 3 vols. Berlin: Akademie Verlag; 1978-1984 (PHP $=$ CMG V, 4,1,2). De sanitate tuenda: K. VI, 1-452 (San.Tu.).

10. Kalokaiagathías: Aff. Dig, V, 35, 8 = Elst, 49. "Quella ricerca che, conducendoci all'adquisizione del bene -il quale è anche chiamato il fine della vita — ci rende felici, o beati, o comunque si voglia dire»: Donini, Pier Luigi. Tipologia degli errori e loro correzione secondo Galeno. In: Manuli, Paola; Vegetti, Mario, eds. Opere psichologiche di Galeno, Napoli: Bibliopolis; 1988, p. 65-116 (74-75); Nussbaum, Martha C. La terapia del deseo. Teoría y práctica en la ética helenística. Barcelona: Paidós; 2003, p. 37-46.

11. Barnes, Jonathan. Galen on logic and therapy. In: Kudlien, Fridolf; Durling, Richard J., eds. Galen method's of healing. Proceedings of the 1982 Galen Symposium. Leiden: E. J. Brill; 1991, p. 50-102 (56, 93-102). También, García Ballester, n. 3, p. 23-25.

12. Lloyd, Geoffrey E. R. Galen and its contemporaries. In: Hankinson, n. 1, p. 34-48 (42-43). 
con sus pacientes, deben ser virtuosos; tienen, afirmaba, que estar disciplinados, al menos, por la virtud de la templanza ${ }^{13}$.

Sin embargo, con esta perspectiva, que hace de la filosofía un asistente constitutivo del saber o del perfeccionamiento personal ${ }^{14}$, la labor de la medicina puede verse reducida a su tarea curativa, la cual incluso puede aparecer tildada de placebo ${ }^{15}$. La tesis galénica de que el médico excelente es también filósofo, queda entonces limitada; se ve como una táctica propagandística dirigida a ganar la confianza de sus pacientes ${ }^{16}$; un dispositivo argumental en la ácida polémica que mantuvo con las otras escuelas médicas ${ }^{17}$; o bien, por último, como un modelo autorreferencial de adquisición de conocimiento en un medio hostil a la medicina griega ${ }^{18}$. Según expuso, esta circunstancia adversa le condujo a volcarse en la filosofía; el razonamiento filosófico le suministraba reglas que le producían la serenidad necesaria para el estudio: «día tras día permanezco en calma ante cualquier cosa que me suceda» ${ }^{19}$, hasta el punto de haber conseguido orientar su vida sólo por el amor al trabajo ${ }^{20}$. Es una muestra, según Boudon-Millot, de una orientación filosófica ética dirigida a hacer de la vida solo una condición para conseguir la excelencia ${ }^{21}$. Y así es: el proyecto contiene tantas resonancias ascéticas que podrían recordar el ideal estoico o el cristiano ${ }^{22}$.

\footnotetext{
13. Opt. Med., I, 61, 1-5 = Boudon-Millot, 291.

14. «Galeno necesita la ética no sólo para adquirir conocimiento acerca de la bondad y de qué cosa sea la virtud, sino también para ser bueno»: Lloyd, n. 12, p. 40-42.

15. Mattern, Susan. Galen and the rhetoric of healing. Baltimore: Johns Hopkins University Press; 2008, p. 69-97.

16. Eijk, Phillip van der. Aristotle! What a thing for you to say? Galen's engagement with Aristotle and Aristotelians. In: Gill; Whitmarsh; Wilkins, n. 1, p. 261-281 (271); Lloyd, Geoffrey E. R. Scholarship, authority and argument in Galen's Quod animi mores. In: Manuli; Vegetti, n. 10, p. 11-42 (40-42); Mattern, n. 15, p. 138-158; Hankinson, n. 2, p. 8-11; Rocca, Julius. Galen on the brain. Anatomical knowledge and physiological speculation in the second century AD. Leiden: Brill; 2003, p. 1-14 y 49-68.

17. Flemming, Rebecca. Demiurge and emperor in Galen's world of knowledge. In: Gill; Whitmarsch; Wilkins, n. 1, p. 59-84 (72); Hankinson, n. 2, p. 5-8 y 13-14.

18. König, n. 1, p. 40-44, Staden, n. 1, p. 150-156; Boudon-Millot, n. 8, p. 175.

19. Ind. = Boudon-Millot; Jouanna, 16, 10-18.

20. Aff. Dig., V, 43, 9-11 = Elst, 54 (tomado de Boudon-Millot, n. 8, p. 185-188). Como régimen para la sabiduría: Aff. Dig., V, 33, 7-14 = Elst, 48; Ind. = Boudon-Millot; Jouanna, 20, 7-19; 22,11-23,12; véase la introducción de los editores: $p$. XLV-LVIII.

21. Boudon-Millot, n. 8, p. 179-180.

22. Gill, Christopher. Naturalistic psychology in Galen \& stoicism. Oxford: Oxford University Press; 2010, p. 262-277; Flemming, n. 17, p. 80; García Ballester, n. 3, p. 7-8, Nussbaum, n. 10, p.
} 
Pero se echa en falta el cometido de la medicina tan destacado por Galeno. Sin la medicina, las ricas analogías ideadas con la filosofía se ensombrecen: no es modelo para las artes y las actividades, sobre todo de la ética, como hizo Aristóteles, ni es ejemplo de la filosofía como remedio, como aparecía en la filosofía de Platón y de los autores helenísticos o romanos ${ }^{23}$. Tampoco puede parangonarse con ella para emularla como, creemos, Galeno quería lograr cuando advertía de que: «meramente desear la verdad no es suficiente» ${ }^{24}$. Cuando leemos a Galeno hablando de la relación entre la medicina y la filosofía percibimos una antigua alianza que, según él, solo se habría roto en su época por la ambición, indolencia e ineptitud de los médicos. Aunque al reflexionar así estaba censurando a Tésalo, esos caracteres le llevaban a una valoración desesperada de la medicina en Roma, que achacaba repetidamente a la falta de amor por el estudio. Esta negligencia afloraba en el comportamiento de los médicos: se equivocan de continuo, decía, si conocieran la lógica podrían razonar en lugar de gritar, lo que acarrea tan mala reputación a la medicina ${ }^{25}$. Pero no pensaba en una escuela filosófica como remedio. El modelo que estaba evocando es el médico: la degeneración de los tiempos es tal, se quejaba, que se cuestiona la validez del método de Hipócrates y se llega al extremo de despreciar la ejemplaridad de su comportamiento ${ }^{26}$.

La figura de Hipócrates le permitía mostrar su intención en torno a la doctrina ética. Por una parte, lo presentaba como un modelo de conducta consecuente a hacer del amor por el trabajo la más alta de las aspiraciones personales. Por otra, advertía del compromiso adquirido con el estudio de todas las partes de la filosofía, la lógica, la física y también la ética para el ejercicio médico ${ }^{27}$. En efecto, aclaraba que al tener la medicina com-

591-622; Rousselle, Aline. Porneia. Del dominio del cuerpo a la privación sensorial. Del siglo II al siglo IV de la era cristiana. Barcelona: Ediciones Península; 1989, p. 17-38; Zambrano, María. Séneca. Madrid: Ediciones Siruela; 1994, p. 32-51.

23. Grimaudo, Sabrina. Difendere la salute. Igiene e disciplina del soggetto nel De sanitate tuenda di Galeno. Palermo: Bibliopolis; 2006, cap. 4, especialmente p. 104-117; Nussbaum, n. 10, p. 33-73; Vegetti, Mario. L'anima, la città e il corpo. In: Quindice lezioni su Platone. Torino: Einaudi; 2003, p. 132-146.

24. Ars medica, I, 244, tomado de Boudon-Millot, n. 8, p. 176.

25. Barnes, n. 11, p. 56-60; Vegetti, n. 7, p. 67-71; Nutton, Vivian. The life and career of Galen. In: Ancient Medicine. London: Routledge; 2004, p. 216-229 (221-222); García Ballester, n. 3, p. 20-21.

26. Opt. Med., I, 53,1-55, 6 = Boudon-Millot, 283-286.

27. Opt. Med., I, 60,16-61,1 = Boudon-Millot, 291. 
petencias sobre el diagnóstico, pronóstico y tratamiento, necesariamente inspiradas por el ideal de bien, estaba obligada a una reflexión moral. Fue Hipócrates, en definitiva, quien reveló la excelencia moral de la medicina. Así expuesto, no es extraño que encontremos a Galeno preguntándose si los que se declaraban seguidores de Hipócrates no habían leído realmente sus obras o no las habrían entendido, pues no quedaban contagiados del deseo filantrópico de afirmar y desarrollar un hábito (héxis) de dedicación al estudio ${ }^{28}$.

Lejos de considerar a la filosofía la fuente de saber moral, sostenemos que la intención galénica fue recuperar el desempeño de la medicina griega como corpus antropológico y como forma filosófica. Proponemos que aquí se enmarca la apología galénica de Hipócrates; es decir, todo el sentido contenido en concebir la medicina como un saber hondamente filantrópico, capaz de curar al enfermo y de constituirse en un guía ética y cultural para la sociedad, con la misma o mejor competencia que la filosofía en el manejo de los asuntos humanos:

«Es útil para los médicos conocer el lugar [del alma hegemónica] para aplicar los remedios cuando la facultad lógica está dañada; pero los filósofos no utilizan este conocimiento ni para descubrir la diferencia entre las virtudes ni para el entrenamiento mismo. Porque si la parte hegemónica del alma está en la cabeza permitirá que podamos adquirir prudencia y templanza y justicia y virilidad (andreían) con una enseñanza y entrenamiento distintos que si está localizada en el corazón» ${ }^{29}$.

Este apoyo en Hipócrates nos sugiere un objetivo existencial, al ser expuesto por Galeno; lo recrea como guía en la búsqueda del mérito personal y del prestigio de la medicina:

«debemos, primero, ser filósofos, si actuamos así nada se opondrá a que lleguemos a ser semejantes a él o, quizá, mejores, al haber estudiado todo aquello que dejó dicho magníficamente este gran médico y poder aplicarnos a descubrir lo que resta ${ }^{30}$.

28. Opt. Med., I, 55,7-58, 5 = Boudon-Millot, 286-288; Lain Entralgo, Pedro. La medicina hipocrática. Madrid: Alianza; 1987, p. 299-318; Jouanna, Jacques. La lecture de l'éthique hippocratique chez Galien. In: Flashar, Hellmut; Jouanna, Jacques, eds. Médecine et morale dans l'antiquité. Geneva: Vandoeuvres; 1997, p. 211-248 (236- 245).

29. PHP, V , 779, 8-16 = CMG V 4,1,2, 586-588.

30. Opt. Med., I, 62, 17-63 = Boudon-Millot, 292. 
Por otra parte, revela la incertidumbre de dejarse guiar por la filosofía; pues no creía que de ella se pudiera obtener certeza a través de la propia experiencia, como procuraba la medicina hipocrática ${ }^{31}$. Ante estas consideraciones, insistió en utilizar lo que llamó «nuestra filosofía». Con tal denominación se refería a una doctrina de carácter médico, que hacía proceder de Hipócrates y que respaldaba con los saberes de Platón y Aristóteles ${ }^{32}$. Este modelo epistémico nos interesa sobremanera, pues Galeno declaró que su proyecto doctrinal era culminar la unión entre medicina y filosofía, para que con la misma doctrina se pudieran proporcionar, a la vez, la salud física y la moral ${ }^{33}$.

Creemos que concebir este objetivo significa apostar por fundar la filosofía práctica en la psicología médica; de hecho, Galeno afirmó que el descubrimiento de la causa de las afecciones del alma (pasiones) «nos ayuda a comprender qué significa vivir de acuerdo a la naturaleza ${ }^{34}$. En este aserto

31. «Pero los filósofos no la han practicado (la demostración científica) como lo han hecho otros» (geómetras, aritméticos, matemáticos, astrónomos, arquitectos): PHP, V, 653,16-654,9=CMG $\vee 4,1,2,485$. «No hay nada más que discutir puesto que las materias bajo investigación han sido descubiertas por medio del método científico, la disección»: PHP, V, 650, 9-10 =CMG V 4,1,2, 480. Y, también, los capítulos 3, 4 y 5 del libro Vl; el libro IX, por ejemplo, PHP, V, 766, 7-15 = CMG V 4,1,2, 576; Hankinson, Richard J. Galen on the limitations of knowledge. In: Gill; Whitmarsh; Wilkins, n. 1, p. 206-242 (210-219); García Ballester, n. 3, p. 9-10.

32. Hipócrates es la fuente del saber humano [PHP, V 724, 4-726,13 = CMG V 4,1,2, 542-544], o lo es la medicina por el método disectivo, para la aclaración de las partes del alma: PHP, V, 519,10-520, 9 = CMG V 4,1,2, 390. En general, de QAM: capítulos VI (para Platón) y VII (para Aristóteles) y VIII para Hipócrates: IV, 799-805 = García Ballester, 55-68; Flemming, n. 17, p. 59-84, 66-67, 72-81; Donini, Pier Luigi. Psychology. In: Hankinson, n. 1, p. 184-209 (186-189 y 197-199); Lloyd, n. 16, p. 15-32; García Ballester, Luis. Soul and body. Disease of the soul and disease of the body in Galen's medical thought. In: Manuli; Vegetti, n. 10, p. 117-152 (134); Eijk, van der, n. 16, p. 270-272. Tieleman, n. 4, p. 55-59. Manuli, Paola. La passione nel De Placitis Hippocratis et Platonis. In: Manuli; Vegetti, n. 10, p. 185-214 (207-214); Jouanna, Jacques. La notion de nature chez Galien. In: Barnes; Jouanna, n. 4, p. 229-268 (242-262).

33. QAM, IV, 767; 814,13-16 = García Ballester, 27; 83; Vegetti, n. 7, p. 92-98. Nutton, Vivian. Galenic medicine. In: Nutton, n. 25, p. 230- 247 (236). Hankinson, Richard J. Galen's anatomy of the soul. Phronesis. 1991; 36: 197-233 (206-208) y Hankinson, James. Actions and passions: affection, emotion and moral self-management in Galen's philosophical psychology. In: Brunschwig, Jacques; Nussbaum, Martha C. Passions and perceptions, Studies in Hellenistic philosohpy of mind. Proceeding of the fifth Symposium Hellenisticum. Cambridge: Cambridge University Press; 1993, p. 184-222 (186).

34. PHP, V, 470, 6-12 = CMG V 4,1,2, 328; Frede, Michael. Galen's theology. In: Barnes; Jouanna, $\mathrm{n}$. 4, p. 73-126 (77-87; 107-111); Hankinson, Richard J. Body and soul in Galen. In: King, Richard A.H., ed. Common to body and soul: Philosophical approaches to explaining living behaviour in Greco Roman Antiquity. Berlin: Walter de Gruyter; 2006, p. 232-258 (246-253). 
subyace una justificación naturalista, que sustenta la introducción de los estudios médicos en el tratamiento de las cuestiones morales: se trataría de conseguir una "condición permanente, consecuencia de una actividad recta y plena de éxito» ${ }^{35}$. Se basa en un a priori: la existencia de una guía natural para conseguir una vida de excelencia, que Galeno definió como el ajustamiento de la conducta a los dictados de la parte racional y divina del alma. Reproducimos su razonamiento, de resonancias aristotélicas ${ }^{36}$ : si se estuviera dotado por naturaleza de una facultad para la excelencia, ella dirigiría la búsqueda de los medios para conseguirla y orientaría el desarrollo de la vida. La definición dada a la facultad racional contiene esa virtualidad directriz:

«en su ejercicio podremos alcanzar una buena disposición (euexía), que nos procurará más alegría que los placeres esclavos del cuerpo. Pues no tenemos otro medio de diferenciarnos de perros, cerdos, corderos y asnos ${ }^{37}$.

Como se aprecia en la cita, el medio aludido, distintivo de la especie, es una construcción que explica el designio antropológico a través del conocimiento fisiológico. Como punto de partida Galeno atribuyó a la especie humana la capacidad racional como la actividad específica dotada por naturaleza; de esta forma, la humanidad aparecía considerada como la realización más acabada de la generación natural: participaba con el mundo animal de la mortalidad y con los dioses de la racionalidad ${ }^{38}$. El diseño continúa informando los siguientes niveles de la descripción fisiológica. Animalidad y divinidad intervienen en la concepción tripartita de facultades, funciones o actividades orgánicas, con una intención antropológica. Este modelo es el fundamento de la doctrina ética: cada una de las facultades tiene su propio hábito, costumbre, función, su propio éthos ${ }^{39}$. La concupiscible, localizada en el hígado y agente de procesos básicos (nu-

35. Calvo Martínez, José Luis, ed. Introducción. In: Ética a Nicómaco. Madrid: Alianza editorial; 2001, p. 7-43 (15)

36. Donini, n. 10, p. 82-85 y 108-113, como ética teleológica: 74-83 y 85-94; sosteniendo su fundamento en Ética a Nicómaco: 98-103.

37. Pecc. Dig., V, 87,10-14 = Elst, 78 .

38. Protr., I, 1-3, 9-10; 21-22; PHP, V, 472, 5-11 = CMG V 4,1,2, 330.

39. El capítulo 7 del libro $V$ de De Placitis está íntegramente dedicado a la exposición de esta doctrina (tres partes oréticas) basándola en Platón, Aristóteles y Posidonio (PHP, V, 479-508 = CMG V 4,1,2, 336-358); el capítulo 2 del libro IV (PHP, V, 372-377 = CMG V 4,1,2, 244-246). También los capítulos 1 y 2 del libro VI (PHP, V, 505-519= CMG V 4,1,2, 360-372); De Lacy, Phillip. The 
trición, reproducción) mantiene el contenido semántico correspondiente a la animalidad más sujeta o próxima a lo impulsivo ${ }^{40}$. La tímica, situada en el ventrículo izquierdo, asiste en las funciones básicas de la vida y, como parte perceptible de estas, desempeña el conjunto de las emociones ${ }^{41}$. La hegemónica, de localización cerebral, ejerce las funciones motora, sensitiva e intelectual ${ }^{42}$; con esta última, añade a la fisiología del mantenimiento y extensión de la vida el «conocimiento del fin que le pertenece a cada uno según su género de existencia» ${ }^{43}$.

La explicación biológica procedía de la doctrina aristotélica ${ }^{44}$, e interesa aquí subrayar la noción de la naturaleza como fuente inteligente de actividad, que tomó Galeno para la comprensión de la función de las almas en el desarrollo moral: «se ha mostrado que es necesario utilizar la facultad concupiscible, llamada así por los antiguos porque tiende ciegamente a los placeres del cuerpo» ${ }^{45}$. Según esto, pudo sostener que las actividades de esta facultad y la de la tímica aportaban una causalidad orética, desiderativa, y con esta, anclar el progreso moral en la fisiología. En el trascurso de la vida, la actuación sobre estos sensibles indicaría, como se estudiará más adelante, la validez del método de conocimiento aplicado para lograr la vida noble. Él siempre confió en que la paideia produciría una disminución del poder de las emociones en él; desde pequeño, decía, fue alentado y vigilado por su padre en esa tarea: la de liberar su mente de los factores corruptibles de la lascivia, tryphé, y también de las emociones descontroladas, los propios de la energía tímica ${ }^{46}$.

third part of the soul. In: Manuli; Vegetti, n. 10, p. 43-63 (44-47, 48-57, 59); Hankinson, n. 34, p. 205.

40. PHP, V, 519,10-538, $8=$ CMG V 4,1,2, 372-388; De Lacy, n. 39, p. 48.

41. PHP, V, 270, 9-271, 7 = CMG V 4,1,2, 154-156; y del libro III los capítulos 2, 3, y 7 (PHP, V, 293-301; 301-310; 335-348 = CMG V 4,1,2, 174-18; 184-192; 212-222).

42. PHP, V, 348, 10-356,14 = CMG V 4,1,2, 222-230; Jouanna, Jacques. Does Galen have a medical programme for intellectuals and the faculties of the intellect? In: Gill; Whitmarsh; Wilkins, n. 1, p. 190-205 (192-196); Rocca, Julius. Anatomy. In: Hankinson, n. 1, p. 242-262 (247- 256); Donini, n. 32, p. 184-195.

43. Pecc. Dig., V, 77,13-14 = Elst, 72 .

44. Donini, n. 10, p. 98-103; Grimaudo, n. 23, p. 35-71; Nussbaum, n. 8, p. 115-125. Eijk, van der, n. 16, p. 266-269; Debru, Armelle. Physiology. In: Hankinson, n. 1, p. 263-282 (265-267).

45. Aff. Dig., V, 27, 12-15 = Elst, 45.

46. Ind. = Boudon-Millot; Jouanna, 18,1-20, 2. 
Si durante el helenismo se elaboró una terapia del deseo analizando las emociones como causas del malestar existencial ${ }^{47}$, Galeno mantuvo la dimensión ascética del proyecto ${ }^{48}$ para conseguir «una forma de vida, un arte de vivir y una manera de ser» con el mismo método ${ }^{49}$. Es decir, pudo haber creído que la verdad, alétheia, podría llegar a convertirse en hábito, ethos: «todo el mundo concuerda en considerar que la falsa opinión en torno al fin conduce a la infelicidad ${ }^{50}$.

\section{Doctrina moral}

Con la incorporación de la medicina al proyecto filosófico, Galeno criticó las tesis epicúreas y estoicas dominantes en la conceptuación, función y significado de las pasiones. La propuesta ética que formuló, fundamentada en la analogía entre enfermedades del alma y enfermedades el cuerpo, supuso activar el cometido moral de la medicina: apaciguar las sacudidas emocionales, que entorpecían la función intelectiva.

La salud fue definida por él como el estado corporal que permite el ejercicio de actividades. Subyacía en esta concepción la idea de que una naturaleza providencial había creado un modelo de organismo dispuesto para una vida de excelencia, es decir, como se ha visto, preparado para la plenitud de la actividad racional ${ }^{51}$. Este supuesto justifica que pudiera estudiar las funciones psíquicas y éticas con el mismo esquema doctrinal que el dedicado al resto de procesos orgánicos, para elaborar una filosofía práctica.

La doctrina parte de la versión del alma tripartita y se realiza en torno al contenido orético de las funciones de cada una de las partes. Como hemos dicho, el deseo lo entendemos en el sentido de despliegue, de expresión de

\footnotetext{
47. Nussbaum, n. 10, p. 36-37 para la enunciación del proyecto.

48. Criterios sensibles que enlazan episteme con axiología: Nussbaum, n. 10, p. 37-46; creemos que también lo entiende así: Hankinson, 1993, n. 33, p. 209-212.

49. Boudon-Millot, n. 8, p. 186.

50. Donini, n. 10, p. 93-94. Foucault, Michel. Discurso y verdad en la antigua Grecia. Barcelona: Paidós; 2004, p. 174-209; Morey, Miguel. Foucault. Tecnologías del yo y otros textos afines. Barcelona: Paidós; 2010, p. 74. Lo entendemos como algo próximo a lo pretendido por la dialéctica aristotélica (Nussbaum, n. 10, p. 81-99); es decir, un conjunto de prácticas para hacer de la verdad un principio permanente de acción (p. 45-46), que reproduce la propuesta de Aristóteles cuando expresaba eudaimonía con eu práttein: Calvo Martínez, n. 35, p. 15.

51. San. Tu., VI, 21, 6-11; Grimaudo, n. 23, p. 59-60.
} 
funciones diseñadas por naturaleza. Cada facultad anímica poseía caracteres simbólicos que alcanzaban irremisiblemente a los aspectos morales de la conducta humana: la parte racional del alma aspira, en la idea galénica, a comprender y conocer y a conseguir la verdad; la tímica desea alcanzar la libertad, la victoria, reputación y honor. La concupiscible pretende la expresión de una gran variedad de actividades vitales, asociadas con placeres corporales $^{52}$.

La función del conjunto de almas dentro del proyecto de vida buena se entiende por medio de la explicación dada a la patología orgánica ${ }^{53}$ : la enfermedad como desequilibrio o asimetría entre las componentes del cuerpo, o de las partes del cuerpo ${ }^{54}$. Con dicha explicación, que Galeno legitimó en Hipócrates, declaró que la etiología del mal existencial ha de explicarse como resultado de un conflicto pulsional entre las partes del alma:

«En muchas ocasiones la razón es tan fuerte sobre la parte tímica del alma que nunca se produce un conflicto entre ellas, la una dirige, la otra es dirigida. Este es el caso de las personas que se han dedicado a la filosofía. En otros casos, la ira es tan fuerte en relación a la razón que llega a dominar y gobernar todo, como es visto que les sucede a muchos bárbaros» ${ }^{55}$.

La analogía proyecta una argumentación poderosa ante la filosofía, que pudo ser calificada como insuficiente o engañosa por él. Le permitió rebatir dos extremos en la definición de las emociones: como actividades somáticas deseables o como percepciones solamente intelectuales. En el primer término desarrolló su crítica a los epicúreos:

52. «Tres son las propiedades (actividades, desempeños) nuestras por naturaleza (oikéiosis), el placer por medio de la desiderativa, la victoria a través de la espirituosa, y la virtud por medio de la lógica». PHP, V, 460, 9-12; 594, 6-13 = CMG V 4,1,2, 318; 432; QAM, IV, 772, 1-14 = García Ballester, p. 329.

53. Creemos que Martha Nussbaum la toma del contexto clínico (n. 10, p. 71-73: «el argumento ha de ser como el estilete», p. 79); y no fisiopatológico como veremos que sostuvo Galeno. Jouanna (n. 43, p. 197) en cambio parece darle una función etiológica a una analogía que no es más que ejemplificadora. Tampoco García Ballester le da un sentido patogénico (n. 3, p. 39).

54. García Ballester, n. 32, 121-124 y 140-142; analogía que le permite a Galeno deslindar las enfermedades del alma: para el filósofo (p. 124); para introducir el concepto platónico (p. 137).

55. PHP, $\vee, 307,7-308,2=C M G \vee 4,1,2,188-190$. En general el libro IX de esta obra recoge esta cuestión: PHP, V, 720-805 = CMG V 4,1,2, 542-608. Aplicado al conocimiento de las enfermedades del alma: PHP, V, 338, 4-6; 775, 1-777, 6 = CMG V 4,1,2, 214; 584-586; 338. 
«Creer como Epicuro que el placer sea un bien es propio de un logos erróneo y falso, y toda la actividad y todos los movimientos particulares del alma que miran perversamente el placer como la finalidad de la vida son necesariamente enfermos y errados, pero no por esto son pasiones» ${ }^{56}$.

Mediante la segunda acepción rechazó la doctrina estoica ${ }^{57}$, representada con Crisipo: considerar que las pasiones y las emociones tienen el carácter de creencias las hace originarias de la facultad racional, y como consecuencia, se entienden como errores del juicio ${ }^{58}$.

Las siguientes palabras nos expresan, quizá retóricamente, el desconcierto que le provocaban estas conjeturas. Por una parte, contra los epicúreos, subrayaba la imposibilidad de asociar deseos opuestos en sus fines, «no se puede concebir el ser amante del trabajo con estar borracho, o de juerga, o dado a los placeres del amor; o por decirlo brevemente, cuando se es esclavo del sexo y del vientre» ${ }^{59}$. Por otra, argumentaba con la observación de los fenómenos corporales que acompañan a las emociones: cómo todo el cuerpo se altera en las afecciones del alma "palidece, se enfría y tiembla, como ocurre en el temor; o enrojece, se calienta y tensa en la inflamada ira» ${ }^{60}$. La intención en este caso era la de refutar la tesis estoica acerca del carácter racional de las pasiones. Imaginando un pensamiento galénico, si uno creyese que la propia pasión se conduce como una creencia, debería poder dominarla con argumentos ${ }^{61}$.

Para demostrar su idea, expuso una reflexión sobre las encontradas impresiones de Medea ante los embates de sus celos, enfáticamente cargadas

56. PHP, V, 372,1-3 = CMG V 4,1,2, 242; Aff. Dig. V. 2,16-3,5= Elst 32; Donini, n. 10, p. 71-74 y $91-$ 94. En esta argumentación referimos las múltiples apelaciones que se han hecho sobre si la indeterminación de la sustancia del alma, que se puede observar a lo largo de la obra galénica, conlleva un retraimiento por parte de Galeno para tratar temas filosóficos. Donini, n. 32; opinamos que no se trata de indeterminación sino de contexto: Singer, Peter N. Levels of explanation in Galen. Classical Quarterly. 1997; 47: 525-542.

57. Sobre el estudio de la plausibilidad y actualidad de la combinación entre ambas doctrinas, estoica y galénica: Gill, n. 22.

58. Nussbaum, n. 10, p. 405-424.

59. Opt. Med., I, 59,11-14 = Boudon-Millot, 290; PHP, V, 456, 3-458, 15 = CMG V 4,1,2, 314-316; Gill, Cristopher. Competing readings of stoic passions. In: The structured self in Hellenistic and Roman thought. Oxford: Oxford University Press; 2009, p. 207-290 (238-266); Hankinson, 1993, n. 33, p. 192-193.

60. PHP, V, 270, 9-19=CMG V $4,1,2,154$. También se reconoce en la estoa, pero sin considerar la modificación cualitativa (con afectación del pneuma): Nussbaum, n. 10, p. 486-487.

61. Nussbaum, n. 10, p. 463-478 y 482-493. 
de sensaciones materiales: si en la facultad hegemónica se dan únicamente las operaciones racionales, «la capacidad de elección y de rechazo, de preparación y de propósito y de asentimiento ¿qué es entonces lo que hace que el alma se ensucie y enturbie?» ${ }^{62}$. La conclusión galénica resume la definición de las pasiones y el valor de las mismas en la filosofía práctica: «lo propio de la pasión es precisamente esto, que el ánima se mueve sin $\log { }^{\prime}{ }^{63}$. Ajeno a la actividad racional, el amor de Medea por Jasón es, para Galeno, un ejemplo del ejercicio desmesurado de la facultad concupiscible, ámetros kinésis toû epithumetikô̂; con esta concepción enuncia también la analogía médica: Medea cometió su crimen «cuando no pudo soportar su ira», a causa de que «estaba dominada por la magnitud de la actividad de sus entrañas (megalósplagnos)» ${ }^{64}$, la razón, doblegada.

Así pues, la conducta alcanza su justo valor semántico e instrumental en el contexto del funcionamiento orgánico, en la explicación de las actividades desarrolladas para la alimentación, la bebida o en la del tiempo dedicado a la vigilia ${ }^{65}$.

«El hábito del alma se corrompe por las malas costumbres en el comer y en el beber y en toda clase de espectáculo. Es necesario conocer las pautas higiénicas de todo esto (...) pues las enfermedades producen pasiones y muchas de ellas dan lugar a una debilidad del calor innato que, en términos higiénicos, es lo primero que hay que guardar» ${ }^{66}$.

Por esta razón, pudo ser esgrimida en el debate filosófico la concepción médica de la enfermedad:

«Si la salud del cuerpo es la proporción entre sus partes simples (...), es decir calor, frialdad, sequedad y humedad, supongo que la salud del alma será la proporción entre sus partes más simples. Tú, [Crisipo] si quieres cumplir con toda tu promesa [la curación de las afecciones del alma] debes desarrollar cuáles son estas partes, cuántas y cómo se relacionan entre sí, pues la enfer-

62. «(...) Nada, sino sus decisiones erróneas. Por esto decimos que la impureza de un alma es un mal juicio y que la purificación consiste en originar una clase de juicio apropiada», pone Séneca en boca de Medea: Braicovich, Rodrigo S. Freedom and determinism in Epictetus' discourses. Classical Quarterly. 2010; 60: 202-220 (214-220).

63. PHP, V, 367,17-369, 7; 372, 1-3 = CMG V 4,1,2, 240; 242; Donini, n. 10, p. 67-72 y 91. Por eso la causa fue una dynamis distinta a la racional (logística): Manuli, n. 32, p. 197 y 202-204.

64. PHP, V, 317,1-318, $10=$ CMG V 4,1,2,196-198.

65. San. Tu. VI, 27,15-28,12.

66. San. Tu. VI, 40,50-41,1. 
medad del alma es una desproporción y un conflicto mutuo (stásis) entre las partes que mantienen la salud del alma» ${ }^{67}$.

Ciertamente, las pasiones son, según Galeno, funciones corporales con deseo de realización, no son errores de juicio; su carácter de impulsos naturales fundamentan el uso del saber fisiológico para realizar la propuesta de modelo educativo.

«La mayor o menor sagacidad o necedad en la parte lógica [del alma] depende de la complexión humoral, la cual, a su vez, depende de la primera generación y de una norma de vida que procure un buen estado humoral, estando ambas cosas íntimamente unidas. Una complexión humoral cálida vuelve irascible y la irascibilidad inflama el calor innato; los que tienen una complexión humoral equilibrada tienen los movimientos del alma moderados» ${ }^{68}$.

El programa presupone que los hábitos y el tiempo moderarían los movimientos afectivos de las partes irracionales del alma, hasta conseguir que obedeciesen totalmente a la razón ${ }^{69}$.

La idea de moldear durante toda la vida el carácter para adquirir la verdad, había sido una creación genuinamente socrática; el hecho de que Galeno la incorporase a su doctrina hasta hacerla un precepto básico, constituía asimismo una actitud común en las corrientes filosóficas de su época ${ }^{70}$. Por tanto, no nos resulta extraño que fuera general el interés por la medicina dietética, y que pudiese ser entendido en un sentido parecido al declarado por él: «en la medida en que llama a la facultad de cada uno a dominar sus apetitos y a practicar la templanza ${ }^{71}$. Pero, en su obra, revela también el malestar ante la depravación que percibía en la sociedad romana: «Si la incorrección debida a la cólera hace del hombre un espectáculo vergonzoso, sucede lo mismo con las del erotismo, de la glotonería, de la embriaguez (...) que son efectos irracionales del alma concupiscible» ${ }^{72}$.

\footnotetext{
67. PHP, V, 440, 37-441, $10=C M G \vee 4,1,2,302$.

68. $Q A M, I V, 807,17-808,12 ; 821,2-9=$ García Ballester 72-73; 86.

69. PHP, V, 426, 1-4; 475, 4-12 = CMG V, 4,1,2, 290; 332. «No me angustio por perder bienes si me queda lo suficiente para no pasar hambre, ni sed; tampoco por los dolores si no me impiden conversar con un amigo o leer un libro»: Ind. = Boudon-Millot; Jouanna, 24, 2-8.

70. Fundamentalmente la de la estoa: Gill, n. 59, p. 286.

71. Opt. Med., I. 56, 17 = Boudon-Millot, 287. Igualmente, De Lacy considera que la tercera parte del alma es el principal objetivo para Galeno, como médico y como moralista (n. 39, p. 59-61); Grimaudo, n. 23, 159-187.

72. Zambrano, n. 22, p. 37-42.
} 
Y este disgusto se une al descrédito de la medicina para demostrarle la insuficiencia de la técnica moral de ascesis, y reafirmar la necesidad de la aplicación de la doctrina médica:

«Si se quiere fijar la mejor conducta del varón, deben ser hechas dos cosas (...) adquirir conocimiento de la verdad y debilitar los movimientos de los afectos por habituación a las buenas prácticas. Así, desde el comienzo se debe moldear el varón con vistas a lo mejor, atendiendo antes que nada a las mismas semillas, y cuidando el régimen de la embarazada, en los alimentos y las bebidas, el ejercicio y el descanso, el sueño y la vigilia; en el deseo y la ira ${ }^{73}$.

Este proyecto concreta, para nosotros, el entramado de la moralidad según Galeno: el impedir un desarrollo desmesurado del funcionamiento de las almas emocional y concupiscible, lo cual acabaría afectando al instrumento de la racionalidad ${ }^{74}$.

Nuestra interpretación mantiene algunas diferencias con otros estudios, utilizados en este trabajo, referentes a la relación entre la higiene del cuerpo y la virtud del alma. Se resaltan los efectos beneficiosos de la contención de costumbres para el desarrollo de una vida virtuosa; concretamente, por medio de la alimentación y la bebida se podía mejorar la memoria y con ello, las condiciones intelectuales ${ }^{75}$. El régimen de vida también era relevante en el contexto de la prevención de la enfermedad mental ${ }^{76}$. Esta interacción se ve reafirmada también antitéticamente; por ejemplo, García Ballester

73. PHP, V, 464, 4-465, $8=$ CMG V 4,1,2, 322 .

74. «Evitar el hambre y la sed, (...) y los baños, y la ira, y las preocupaciones, y las penas y todo lo que pueda decirse en cada circunstancia que modifique el temperamento, krâsis»: San. Tu., VI; 28, 6-11; QAM, IV, 821-822 = García Ballester, 86; Aff. Dig., V, 45, 2-46, 10 = Elst, 55. Para García Ballester, n. 32, Galeno une las virtudes del alma a los temperamentos del cuerpo (p. 128-133). Para Donini (n. 32, p. 196-198) este tipo de acercamiento le habría permitido, a Galeno, la intervención de la medicina en el marco de la creación de una naturaleza adecuada. Hankinson, 1993, n. 33, p. 198-203 y 206-209 lo desarrolla, en cambio, dentro de un modelo cognitivo.

75. Lloyd, n. 16, p. 27-28, 32-37 y 39. No nos resultan convincentes las explicaciones basadas en: impedir el desarrollo de la enfermedad mental (p. 20 y 32); diferenciar durante la infancia por las aptitudes (p. 33 y 37), o el papel de la memoria en la educación (p. 20-24). Por otra parte, no se consideran en este trabajo los aspectos deontológicos con los que se suele reconocer la ética médica: «conjunto de reglas que gobiernan la relación médico-paciente que incorporan un juicio de valor». Pigeaud, J. Les fondaments philosophiques de l'éthique médicale: le cas de Rome. In: Flashar; Jouanna, eds., n. 28, 254-296 (256).

76. Jouanna, n. 42; Gill, n. 22 p. 315-329. 
destacó que la salud corporal podría verse afectada por las disposiciones morales viciosas y la tendencia a actuar bajo el dominio de las emociones ${ }^{77}$. Pero resulta infructuoso el diferenciar la ética de otros aspectos del funcionamiento humano en el mundo clásico: la incorrección moral era para Galeno enfermedad, que perjudicaba la capacidad de la razón. Creemos, para terminar, que en este marco conceptual estaba incluido el compromiso con la excelencia y el prestigio de la medicina.

\section{Medicina y ética}

La filosofía práctica de Galeno, al mismo tiempo que justificaba el ideal de la contención, acogía sus invectivas contra la sociedad romana: «de esta manera, no supuso para mí una gran aflicción la pérdida de toda clase de bienes (en el incendio del templo de la paz), sino la permanencia en la corte imperial, que yo no deseaba» ${ }^{78}$. Es una declaración en la que se aprecia la concurrencia de motivaciones y razonamiento en el desarrollo de una actitud ética, que, como se señalaba en la introducción, se vincula con la epistemología médica; es decir, se explica que la ética se nutre de la trama del razonamiento conjetural: la experiencia moral constituiría el campo de reflexión acerca de la validez del método. Sin embargo, creemos que el ámbito experiencial fue además de una forma de conciencia, utilizado epistemológicamente, como portador de cualidades sensoriales para el razonamiento. En el debate filosófico en torno a la actuación sobre las emociones y las pasiones en el desarrollo del proyecto existencial (si hacerlas desaparecer o dominarlas) Galeno eligió el ejercicio del dominio: «el control de esta pasión (la concupiscible) no consiste en procurarle los bienes que desea, pues se fortalecería, sino que, reprimiéndola, se empequeñece y debilita, de tal suerte que acaba sometida a la razón por su flaqueza» ${ }^{79}$.

Este planteamiento permite que el postulado teórico alcance una expresión perceptible, de alcance epistemológico relevante para devenir el ser

77. García Ballester, n. 3, p. 10-12 y García Ballester, n. 32, p. 150-152. Grimaudo, n. 23, p. 159-189; Eijk, n. 4, p. 299-300.

78. Y continúa criticando el deseo de una riqueza mayor de la necesaria para suprimir el hambre, la sed o el frío: Ind. = Boudon-Millot; Jouanna, 24, 13-25, 12.

79. Aff. Dig., V, 28, 6-9 = Elst, 45. También Ind. = Boudon-Millot; Jouanna, 21, 5-23, 5 . 
acabado, téleios anér, que había ideado ${ }^{80}$. Nos referimos a los límites de la propia inspiración racional que Galeno situó como guía moral. Puesto que la excelencia era para él una mera consecuencia de seguir los dictados de la razón, la cuestión sobre la elección vital llega a parecer agónica: « ¿qué condiciones deberían darse para asegurar estar en la verdad (...), para impedir caer en la desesperanza de encontrarla?» Entre las que procedió a indicar podemos observar que algunas auguran el logro: el entrenamiento y la educación temprana, desde la niñez; el firme esfuerzo para hacer del estudio la única atracción de día y de noche; el hacerse de un método y practicarlo durante toda la vida, «dadas estas condiciones, ¿qué impediría que la búsqueda de la verdad alcanzase el éxito»? ${ }^{81}$ La metáfora médica podía alimentar la certidumbre:

«Sea el lógos sano y veraz (...) el que se mueve bajo su guía no caerá jamás víctima de las pasiones. Guiado por el logos veraz actuará de un modo conveniente y correcto, guiado por el falso, sus movimientos serán malos y errados» ${ }^{82}$.

El trasfondo semántico de este paradigma ético aporta su propia garantía epistemológica, al introducir las representaciones en torno a los cuerpos. En primer lugar, nada de esta aspiración (conseguir la verdad, la serenidad, el vivir según el conocimiento: áriste paideía) era posible para los que por naturaleza no estaban dispuestos para la virilidad (andreían) ${ }^{83}$. El carácter viril, además de su expresión simbólica, dotaba al programa ascético de criterios sensibles y de nociones tradicionales. El control discurría el conflicto personal y la diferencia social: «porque con gran justicia lo superior por naturaleza gobierna y se impone en todas las cosas: el jinete sobre el

\footnotetext{
80. Aff. Dig., V, 14, 11-12 = Elst, 38 .

81. On the composition of art of Medicine, CMG V.1.3, 71, 23-72, tomado de Boudon-Millot, n. 8, p. 176; Hankinson, 1993, n. 33, p. 204-209.

82. PHP, V, 387, 12-16 = CMG $, \mathrm{V} 4,1,2,257$. «El conocimiento es como un monarca y un déspota y nadie se equivoca cuando está presente; los errores son cometidos por los que han sido persuadidos o han olvidado o han sido forzados (...) pero esto no es una afección, como tampoco lo es el no tener conocimiento»: PHP, V, 407, 10-408, $1=C M G ~ V ~ 4,1,2,272-274$.

83. Ind. = Boudon-Millot; Jouanna, 18,18-19. Moreno Rodríguez, Rosa M. La ideación científica del ser mujer. Uso metafórico en la doctrina galénica. Dynamis.1995; 15: 103-149. La relación entre el desarrollo del alma tímica y la virilidad connota magnanimidad y grandeza; define diferencias entre varones y mujeres, pero efectúa también una discriminación moral entre una cualidad, la grandeza de ánimo, y su defecto: dota a la primera de la capacidad de apreciación del mundo de los valores. Manuli, n. 32, p. 190-191.
} 
caballo; el cazador sobre su pieza; la razón sobre la ira» ${ }^{84}$. En definitiva podía acoger todo tipo de comparaciones en torno a la excelencia ${ }^{85}$ : «el alma de la multitud de los hombres vulgares (phaúloi) [son iguales] a los cuerpos que enferman por poca cosa; las almas de los poseídos por la cólera y la ira (orgizoménon y thumouménon) (...) a los cuerpos ya enfermos» ${ }^{86}$.

Es decir, esta idea, un proyecto vital en el que el ejercicio de control dejará emerger o desarrollarse libremente a la racionalidad ${ }^{87}$, posibilitó el desarrollo de una doctrina médica acerca de la función de la templanza, sophrosúne, en la salud humana y en el orden social. En ambos modelos, dietético y ético, subyace discursivamente una disciplina, la vigilancia de hábitos, lo que significa que la dieta, el régimen de vida, contiene en sí misma la preceptiva moral: «las almas de los que se ejercitan en la virtud son iguales a los cuerpos de las personas robustas ${ }^{88}$. En el caso de conducta moral la observación debía ser realizada por tutores, de reconocida virtud, a quienes se les encomendaba taxativamente la censura de las conductas incorrectas durante toda la vida ${ }^{89}$. El amortiguamiento de las sensaciones corporales ligadas a las pasiones supondría, al fin, el haber interiorizado la tutela a modo de componente histórico: «se trata de tener siempre presente la fealdad interior de los que montan en cólera y la belleza de los que se abstienen de caer en la ira» ${ }^{90}$.

Por último, la incontinencia y el desequilibrio entre las partes especifican también la etiología del mal que afectaba a la medicina:

«Se comportan [los médicos] despóticamente con sus colegas y discípulos y dejan que sus pacientes los traten como esclavos; este comportamiento es

\footnotetext{
84. PHP, V, 303,1-3 = CMG V 4,1,2,184.

85. Aff. Dig., V, $28,11-16=$ Elst, 45 .

86. PHP, V , 434, 11-13 = CMG V 4,1,2, 296; hasta extender la analogía desde la naturaleza vegetal y animal a la sociedad: PHP, V, 434, 13-442, 11 = CMG V 4,1,2, 296-302.

87. PHP, $\vee, 483,1-486,6=C M G \vee 4,1,2,338-342$. No hay tal cosa como autocontrol o moderación si el poder desiderativo se elimina de nuestra teoría: PHP, V, 486, 4-6 = CMG V 4,1,2, 342; solo quedaría la prudencia como virtud si no se contase con la desiderativa y la espiritual: PHP, $\vee, 489,8-10=C M G, V 4,1,2,430$

88. PHP, V, 433, 4-6 = CMG V 4,1,2, 296.

89. Aff. Dig., V, 7,10-21, $5=$ Elst, 31-41.

90. «A fuerza de ver las bellas acciones de mi padre en oposición a las fuertes pasiones de mi madre concebí seguir unas y huir y aborrecer las otras»: Aff. Dig. V, 41, 2-5 = Elst, 52. La experiencia es el lugar propio del juicio práctico: Nussbaum, n. 10, p. 99; «The excellence of our way of life guarantees the supremacy of our methodos, the bios being some sort of a reflection of the methodos»: Boudon-Millot, n. 8, p. 183.
} 
opuesto al de los antiguos hijos de Esculapio, que enseñaron a regir a sus pacientes como el general gobierna a sus soldados y el monarca sus asuntos» ${ }^{91}$.

Esta analogía manifiesta el pensamiento galénico: el desorden, la desubicación, el desequilibrio como inmoralidad y ésta como enfermedad ponzoñosa para la razón: «todo lo bello se corromperá, nadie podrá acceder nunca a la verdad, nadie se esforzará en aprender el método lógico, al preferir la búsqueda de la gloria y el poder político» ${ }^{92}$.

En la obra de Galeno, la figura de Hipócrates se convierte en la justificación de los comportamientos ideales morales y funda en el conocimiento médico el desarrollo de la excelencia:

«Según el filósofo sólo hay que modelar las costumbres del alma, pero el médico debe atender el cuerpo (...) La ira, el llanto, la cólera, el dolor, una preocupación innecesaria o el insomnio desarrollan en los cuerpos la fiebre y originan grandes enfermedades. De este modo, se producen pereza del pensamiento y necedad» ${ }^{93}$.

Estas palabras de Galeno nos parecen suficientemente elocuentes para concluir el trabajo. Con ellas justifica la intervención de la medicina en el tratamiento de la conducta humana, al proponer una etiología y una patogenia de carácter médico que clarifican la relación entre las aspiraciones racionales y el modo de vida.

91. Tomado de Barnes, n. 11, p. 58.

92. Tomado de Vegetti, n. 7, p. 69.

93. San. Tu., VI, 40, 8-17. 


\section{Ethics and medicine in Galen's work}

Rosa M. Moreno Rodríguez .................................

1.--Introduction. 2.-Philosophy and medicine. 3.-Practical philosophy. 4.Medicine and ethics.

ABSTRACT: In this study, I propose that Galenic ethics need to be understood within the context of medical doctrine. Recently, published work on the relations among Galen's self-fashioning programme, medical methodology and championing of Hippocrates has supported this hypothesis. I emphasize the idea that medical doctrine was an explanation of the concept of a good human life, a tool to be used in furthering the fulfilment of this objective and indeed one of the purposes of medicine. The basis of my argument is that practical philosophy was devised through a biological approach that attested to the existence of an 
organic purpose for those activities with a moral meaning (corporal desires, emotions, passions). This idea legitimizes the intervention of medicine in the realm of human behaviour. If emotions and desires have a physiological role it is surely the concern of medicine to explain them, and it will also, following the theoretical procedures established for dealing with any malfunction, effect a diagnosis and suggest treatment if either or both are unsound. Furthermore, one of the traditional ideas of disease, the imbalance between parts, explains immoral conduct without recourse to reason. In this way reason becomes a sure guide in the formation of character. According to this view, Galen, and by extension medicine, exert an authority within the debate over the causes of the frequent crises in the Roman world, well over and above (in Galen's own words) that offered by philosophy. When Galen proposed an interpretation of existential distress, anxiety and the persistence of evil as imbalances among organic parts, he was alluding to the breaking-up of the structural organization of the body and suggested that the contemporary milieu, which he considered reprehensible, could be attributed by analogy to the disorder in the structure of Roman society. 